\title{
Overexpression of miR-101 promotes TRAIL-induced mitochondrial apoptosis in papillary thyroid carcinoma by targeting c-met and MCL-1
}

\author{
Jie Zhu' ${ }^{1}$ and Zhenjie $\mathbf{L i}^{1}$ \\ ${ }^{1}$ Department of Endocrinology, Linyi People's Hospital, Linyi, China, 276000 \\ Correspondence to: Zhenjie Li, email: zmkqtn526@yeah.net \\ Keywords: papillary thyroid carcinoma; miR-101; TRAIL; C-met; MCL-1 \\ Received: July 26, $2017 \quad$ Accepted: August 28, $2017 \quad$ Published: September 23, 2017 \\ Copyright: Zhu et al. This is an open-access article distributed under the terms of the Creative Commons Attribution License 3.0 \\ (CC BY 3.0), which permits unrestricted use, distribution, and reproduction in any medium, provided the original author and source \\ are credited.
}

\section{ABSTRACT}

Tumor necrosis factor-related apoptosis inducing ligand (TRAIL) induces apoptosis in malignant cells, but not in normal cells. As papillary thyroid carcinoma cells broadly expressed TRAIL receptors (death receptor 4 and death receptor 5 ) on their surface, TRAIL is considered as a promising drug for treatment of papillary thyroid carcinoma. However, resistance to TRAIL still be a big obstacle to achieve a satisfactory effect for cancer therapy. Here, we found that overexpression of miR101 was able to sensitize papillary thyroid carcinoma cells to TRAIL treatment in vitro and in vivo. Mechanically, we found that genes of c-met and MCL-1 were the targets of miR-101. Overexpression of miR-101 in TPC-1 significantly decreased the cellular protein levels of c-met and MCL-1, and thus inhibiting the PI3K/AKT pathway and reducing the resistance to TRAIL-induced mitochondrial apoptosis. Enforced expression of either c-met or MCL-1 could partially inhibit the miR-101 promoted apoptosis in TRAIL-treated TPC-1 cells. These results indicated that miR-101-c-met/ MCL-1 axis determined the sensitivity of TRAIL to thyroid cancer in some extent. Combination with TRAIL and miR-101 may represent a novel approach to kill papillary thyroid carcinoma cells efficiently.

\section{INTRODUCTION}

Papillary thyroid carcinoma (PTC) represents as the most common malignancy of the endocrine system, which is accounting for about $90 \%$ of the whole thyroid carcinoma cases $[1,2]$. Although prognosis for patients with PTC is often favorable after surgical resection, about $10-15 \%$ of PTC tumors recur and occur distant metastasis $[3,4]$. For these patients, systematic treatment is required. Unfortunately, PTC cells usually exhibited low sensitivity to chemo- or radio- therapy $[5,6]$.

Tumor necrosis factor-related apoptosis inducing ligand (TRAIL) binds to death receptor 4 (DR4) and death receptor 5 (DR5) which exists on cell surface, and then triggers caspase-8-dependent extrinsic and mitochondrial apoptosis $[7,8]$. Selectively, TRAIL induces apoptosis in malignant cells, but not in normal cells. Furthermore, as papillary thyroid carcinoma cells broadly expressed DR4 and DR5 on their surface, TRAIL is considered as a promising drug for treatment of PTC $[9,10,11]$. However, some cancer cells develop mechanisms against TRAILmediated apoptosis $[12,13]$. It's urgent to identify the mechanisms and sensitize PTC cells to TRAIL-mediated apoptosis to achieve a satisfactory effect for cancer therapy.

MicroRNAs (miRNAs) are a class of endogenous and non-coding RNA oligonucleotides with 19-25 nucleotides. MiRNAs act as negative regulators of gene expression through binding to the complementary sequences of the 3'untranslated region (3' UTR) of their target genes $[14,15]$. It has been reported that about $60 \%$ of human genes are regulated by miRNAs. Furthermore, over $50 \%$ of these genes participate in cancer proliferation, metastasis and apoptosis $[16,17]$. It is clear that miRNAs 
play important roles in cancer biological processes. Recently, studies demonstrate that aberrant expression of miRNAs in cancers is responsible for resistance to various anti-tumor drugs including TRAIL [18, 19, 20]. In the present study, we found that expression of miR-101 was decreased in PTC cells. Overexpression of miR-101 was able to sensitize PTC cells to TRAIL treatment in vitro and in vivo by targeting c-met and MCL-1.

\section{RESULTS}

\section{Overexpression of miR-101 sensitizes PTC cells to TRAIL-induced cell death}

To explore the potential role of miR-101 in PTC, we first detected the expression of miR-101 in human thyroid epithelial cell line Nthy-ori3-1 and PTC cell lines (TPC1, BCPAP and K1). We observed that expression level of miR-101 in PTC cells was significantly lower than that in Nthy-ori3-1 cells (Figure 1A). It suggested that aberrant expression of miR-101 was required in PTC. To study the potential benefit of miR-101 to sensitize PTC cells to TRAIL, we changed the cellular level of miR-101 by transfecting with miR-101 mimics or inhibitors (Figure 1B) before they were treated with TRAIL. In addition, the dose dependency of TRAIL to these PTC cancer cell lines was shown in Figure 1C. We chose the concentration of $2 \mathrm{ng} / \mathrm{ml}$ TRAIL which induced slight cell death of PTC cells for combination treatment with miR-101 mimics or inhibitors. Interestingly, we found that overexpression of miR-101 sensitizes PTC cells to TRAIL-induced cell death, whereas the anti-miR-101 reduced the cytotoxicity of TRAIL to these PTC cells (Figure 1C). Since sensitivity of TPC-1 to TRAIL single treatment was in the middle hierarchy, and the TRAIL-induced cell death in TPC-1 can be dramatically augmented, we performed our following experiments to examine the miR-101-induced changes in this PTC cell line.

\section{MiR-101 targets c-met and MCL-1 in PTC cells}

To understand the mechanisms by which miR101 sensitizes TRAIL-induced cell death in PTC, public databases (TargetScan, miRanda, and PicTar) were used to predict the targets of miR-101. Among the candidates, genes of c-met and MCL-1, which have been reported to be associated with TRAIL sensitivity to several cancers $[21,22]$, were commonly predicted by all of these databases and contain complementary sequences paired with miR-101 at the 3' UTR (Figure 2A). Furthermore, contrary to decrease of miR-101 levels in PTC cells, expression of c-met and MCL-1 in PTC cells was obviously overexpressed compared to the human thyroid epithelial cell line Nthy-ori3-1 (Figure 2B). These results suggested the negative correlation between miR-101 and c-met/MCL-1. To confirm that
miR-101 targeted c-met and MCL-1 in PTC cells, we detected the protein levels of c-met and MCL-1 in TPC-1 cells after they were treated with miR-101 and TRAIL. We found that overexpression of miR-101 significantly decreased the expression of both c-met and MCL-1 in TRAIL-treated PTC-1 cells (Figure 2C). Furthermore, results of luciferase reporter assays showed that miR101 overexpression was able to shown to decrease the luciferase activity of pGL3 reporters with wild c-met or MCL-1 3' UTR, while it failed to repress the pGL3 reporters with mutant c-met or MCL-1 3' UTR in TPC-1 cells (Figure 2D). Taken together, we prove that miR-101 targeted c-met and MCL-1 in PTC cells.

\section{Expression of c-met and MCL-1 is associated with TRAIL sensitivity to PTC}

Since previous studies have demonstrated that c-met and MCL-1 regulate TRAIL sensitivity to several cancers $[21,22]$, we next investigated whether expression levels of c-met and MCL-1 were associated with TRAIL sensitivity to our PTC cell line TPC-1. We therefore performed gainand loss-of-function experiments on c-met and MCL-1 by transfecting with eukaryotic expression vector and small interfering RNA (siRNA), respectively. Transfection efficiency of c-met and MCL-1 plasmid and siRNA was shown in Figure 3A. We found that overexpression of either c-met or MCL-1 partially reduced the TRAILinduced cell death in TPC-1. On the contrary, knockdown of either c-met or MCL-1 significantly sensitized the TPC1 cells to TRAIL-induced cell death (Figure 3B). However, gain- and loss-of-function of c-met and MCL-1 failed to change the TRAIL-induced activation of caspase-8 in TPC-1 (Figure 3C). These results suggested that c-met and MCL-1 negatively regulated the TRAIL-induced cell death in PTC through inhibiting the downstream of caspase- 8 pathway.

\section{MiR-101 sensitizes TPC-1 cells to TRAIL via down-regulating the expression of c-met and MCL-1}

As miR-101 targets c-met and MCL-1 in PTC, we next studied the role of c-met and MCL-1 in miR-101promoted cell death in TPC-1 cells. We showed that transfection with c-met and MCL-1 plasmid abolished the effect of miR-101 on suppressing the expression of c-met and MCL-1, respectively (Figure 4A). Moreover, both c-met and MCL-1 plasmid partially inhibited the effect of miR-101 on TRAIL-induced cell death in TPC-1 cells (Figure 4B). In addition, overexpression of miR-101 failed to promote the TRAIL-induced activation of caspase- 8 in TPC-1 cells (Figure 4C). We demonstrated that miR-101 promoted TRAIL-induced cell death in PTC by targeting c-met and MCL-1 which were the downstream regulators in caspase- 8 pathway. 
A

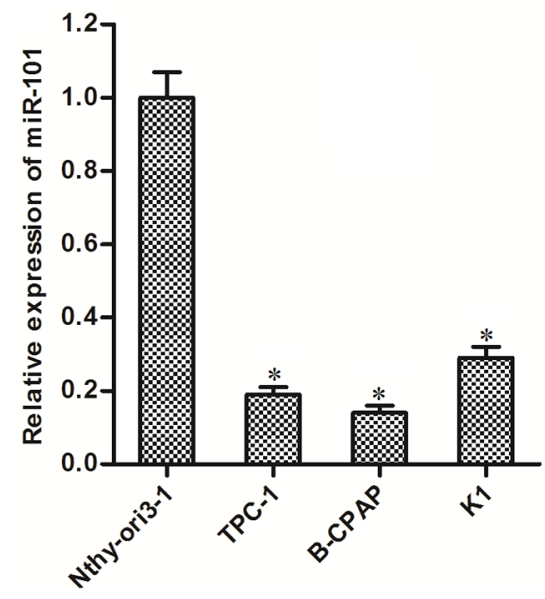

B

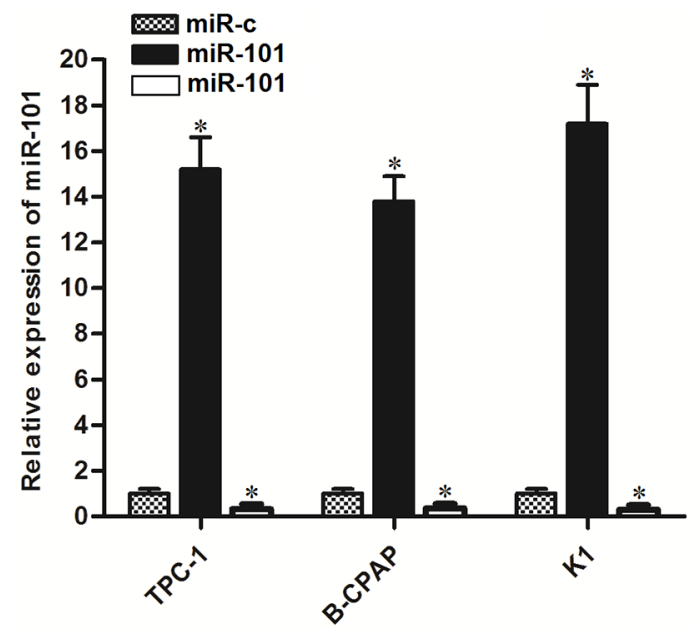

C

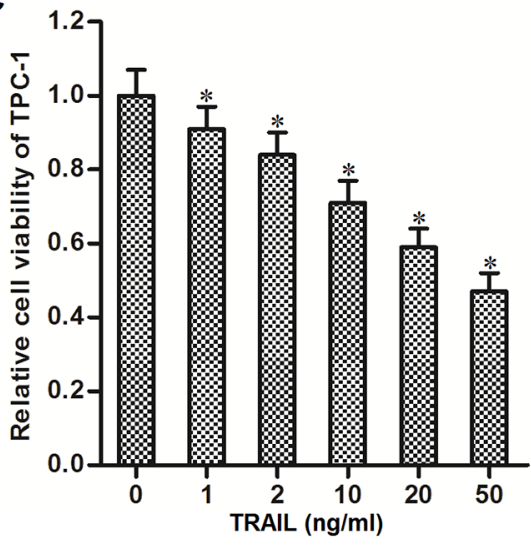

D

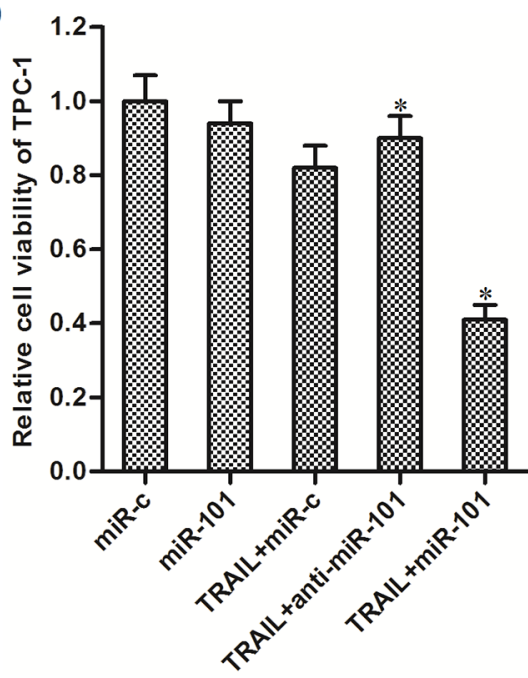

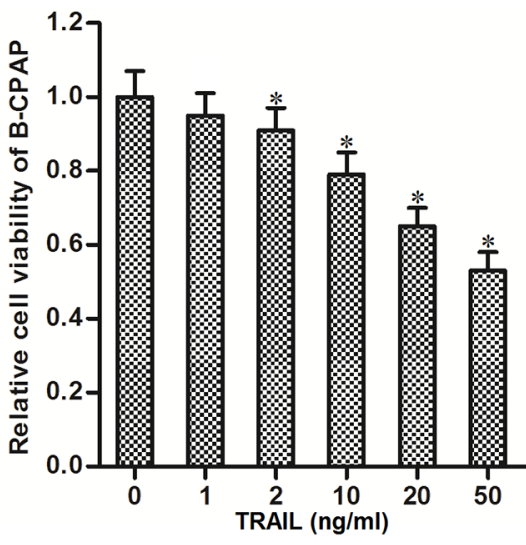
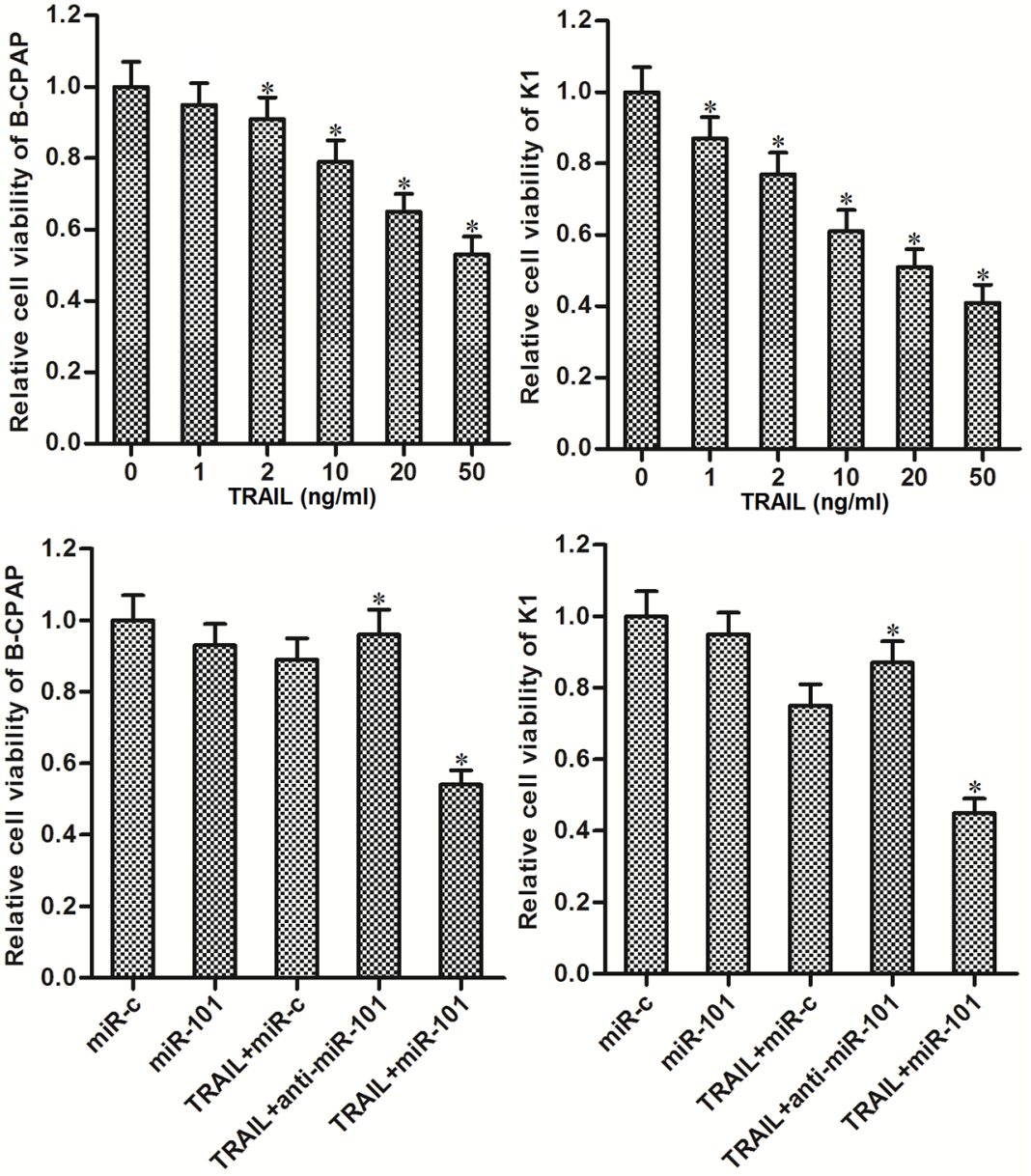

Figure 1: Overexpression of miR-101 sensitizes PTC cells to TRAIL-induced cell death. (A) QRT-PCR analysis was performed to detect the expression level of miR-101 in human thyroid epithelial cell line Nthy-ori3-1 and TPC-1, BCPAP and K1 PTC cell lines. ${ }^{*} P<0.05$ vs. Nthy-ori3-1 cells. (B) Effect of miR-101 mimics or inhibitors transfection on changing cellular level of miR-101 was evaluated by qRT-PCR analysis. ${ }^{*} P<0.05$ vs. miR-c group. (C) TPC-1, BCPAP and K1 cells were treated with different concentrations of TRAIL for $48 \mathrm{~h}$. MTT assays were performed to evaluate the cell viability of them. ${ }^{*} P<0.05 v s$. control group. (D) TPC-1, BCPAP and K1 cells were transfected with miR-101 mimics or inhibitors $(50 \mathrm{pmol} / \mathrm{ml})$ before they were treated with TRAIL $(2 \mathrm{ng} / \mathrm{ml})$. MTT assays were performed to evaluate the cell viability of them. ${ }^{*} P<0.05 v s$. TRAIL+miR-c group. 


\section{Overexpression of miR-101 promotes TRAIL- induced mitochondrial apoptosis in PTC}

Results of western blot analysis showed that overexpression of miR-101 inhibited phosphorylation of PI3K and AKT in TPC-1 cells. As Bad is one of the substrates for AKT [23], we observed that introduction with miR-101 induced dephosphorylation of $\mathrm{Bad}$. However, enforced expression of c-met was found to restore the phosphorylation of PI3K, AKT and its substrate of Bad in miR-101-overexpressed TPC-1 cells (Figure $5 \mathrm{~A})$. These results indicated that overexpression of miR101 induced dephosphorylation of Bad through the c-met/ PI3K/AKT pathway. Dephosphorylated Bad functions as a pro-apoptotic protein via interacting with $\mathrm{Bcl}-\mathrm{xl}$, and thus inactivating it which is a powerful anti-apoptotic protein [24]. As the dephosphorylated Bad was significantly increased in miR-101-overexpressed TPC-1 cells, our results showed that overexpression of miR-101 obviously enhanced the interaction with Bad and Bcl-xl (Figure 5B). Studies have proved that both Bcl-xl and MCL-1 functions as a suppressor in TRAIL-induced mitochondrial apoptosis $[22,25]$. As miR-101 decreased the expression of MCL1 and inactivated the Bcl-xl, we next investigate the role of miR-101 in TRAIL-induced mitochondrial apoptosis in TPC-1 cells. As shown in Figure 5C, overexpression of miR-101 dramatically enhanced the TRAIL-induced collapse of mitochondrial membrane potential (MMP). Furthermore, enforced expression of both c-met and MCL1 partially inhibited the MMP collapse in TRAIL-treated

A

B
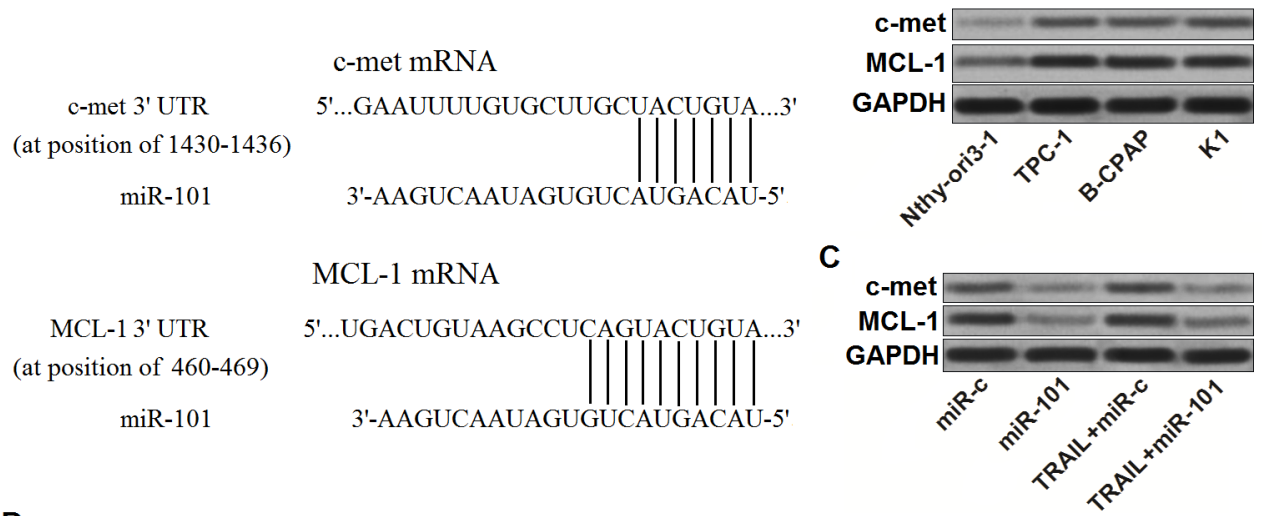

D
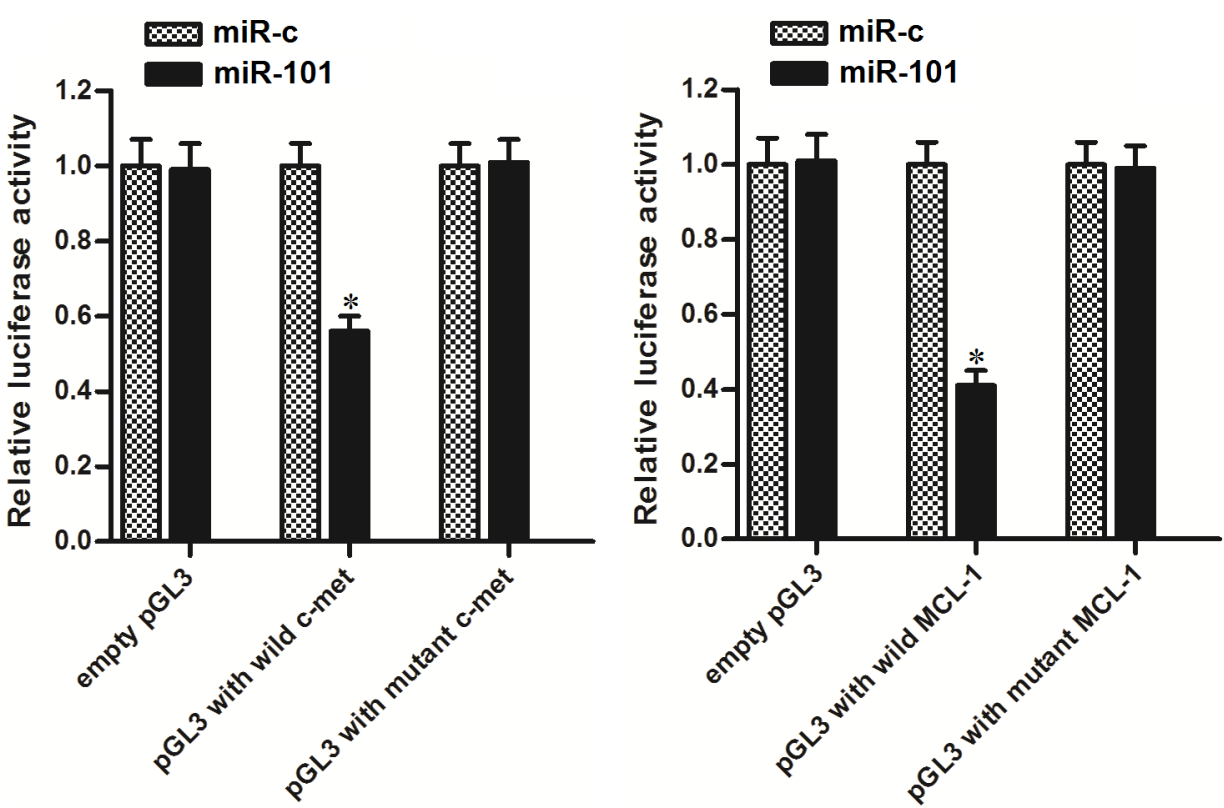

Figure 2: miR-101 targets c-met and MCL-1 in PTC cells. (A) C-met and MCL-1 were predicted as the targets of miR-101 by the public databases of TargetScan, miRanda, and PicTar. (B) Protein levels of c-met and MCL-1 in human thyroid epithelial cell line Nthyori3-1 and TPC-1, BCPAP and K1 PTC cell lines were evaluated by western blot analysis. (C) Protein levels of c-met and MCL-1 in TPC-1 cells were evaluated after they were treated with miR-101 and TRAIL ( $2 \mathrm{ng} / \mathrm{ml})$. (D) Dual-Luciferase Reporter Assay System was used to detect the luciferase activities in TPC-1 cells which were co-transfected with wildt/mutant 3'-UTR of c-met/MCL-1 and miR-101 mimics. ${ }^{*} P<0.05$ vs. miR-c group. 
TPC-1 cells. Due to the damage of mitochondria, the TRAIL- and miR-101-cotreated TPC-1 cells released more amount of cytochrome $\mathrm{c}$ from the mitochondria compared to the TPC-1 cells treated with TRAIL alone (Figure 5D). As cytochrome $\mathrm{c}$ in cytoplasm triggers caspase-9 via inducing Apaf-1/caspase-9 complex [20], we showed that overexpression of miR-101 significantly enhanced the
TRAIL-induced activation of caspase-9 and -3 (Figure 5E) and occurrence of apoptosis (Figure 5F) in TPC-1 cells through inhibiting the expression of c-met and MCL-1. We concluded that overexpression of miR-101 was able to inhibit the expression of c-met and MCL-1, and thus promoting TRAIL-induced mitochondrial apoptosis in PTC.
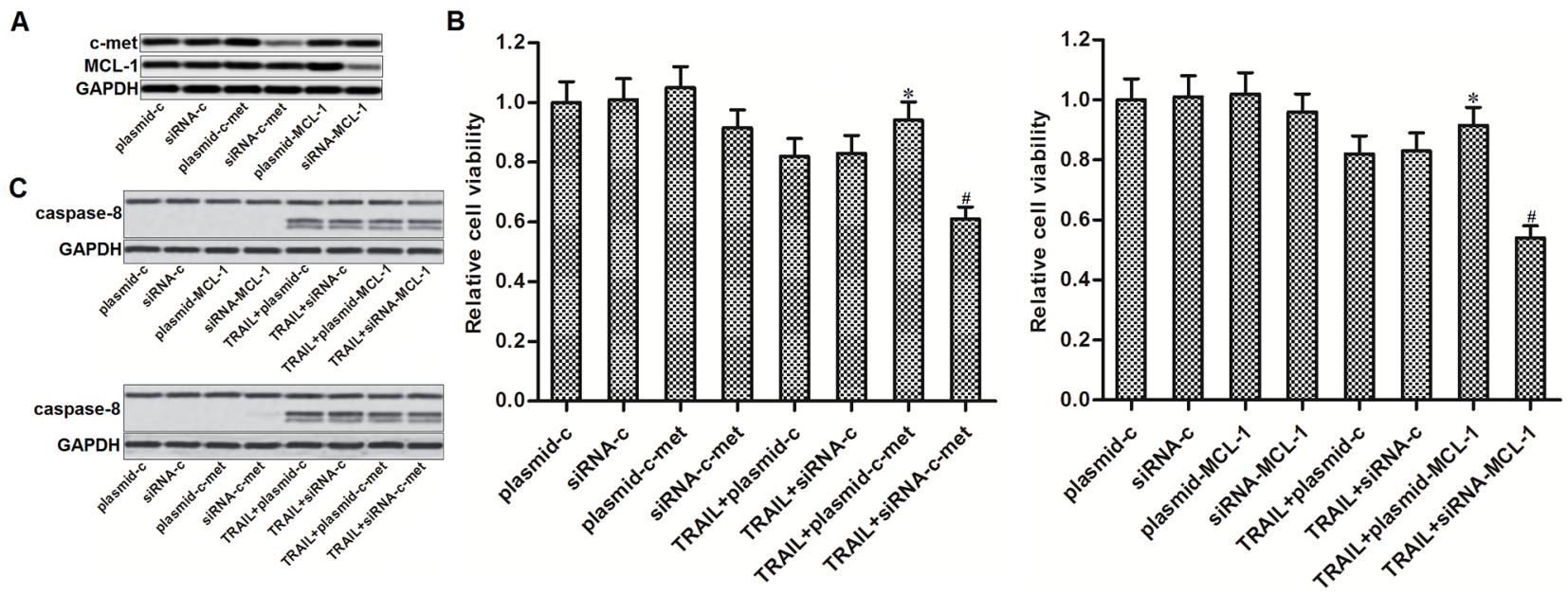

Figure 3: C-met and MCL-1 negatively regulated the TRAIL-induced cell death in PTC. (A) Western blot analysis was performed to evaluate the transfection efficiency of c-met and MCL-1 plasmid and siRNA in TPC-1 cells. (B) MTT assays were performed to evaluate the gain- and loss-of-function of c-met and MCL-1 on TRAIL-induced cell death in TPC-1. ${ }^{*} P<0.05 v s$. TRAIL+plasmid-c group. ${ }^{\#} P<0.05$ vs. TRAIL+siRNA-c group. (C) Western blot analysis was performed to evaluate the gain- and loss-of-function of c-met and MCL-1 on TRAIL-induced activation of caspase- 8 in TPC-1 cells.

A

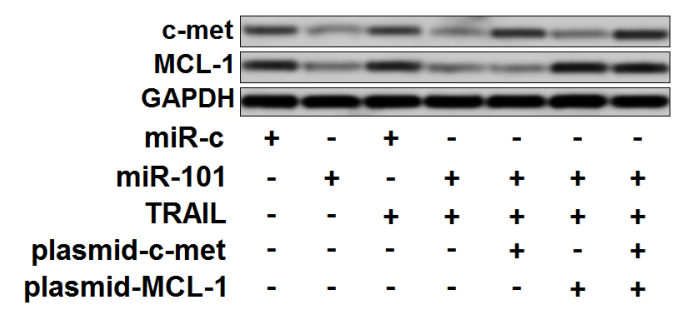

C

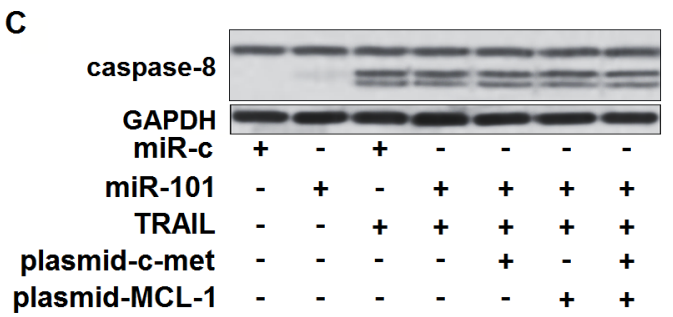

B

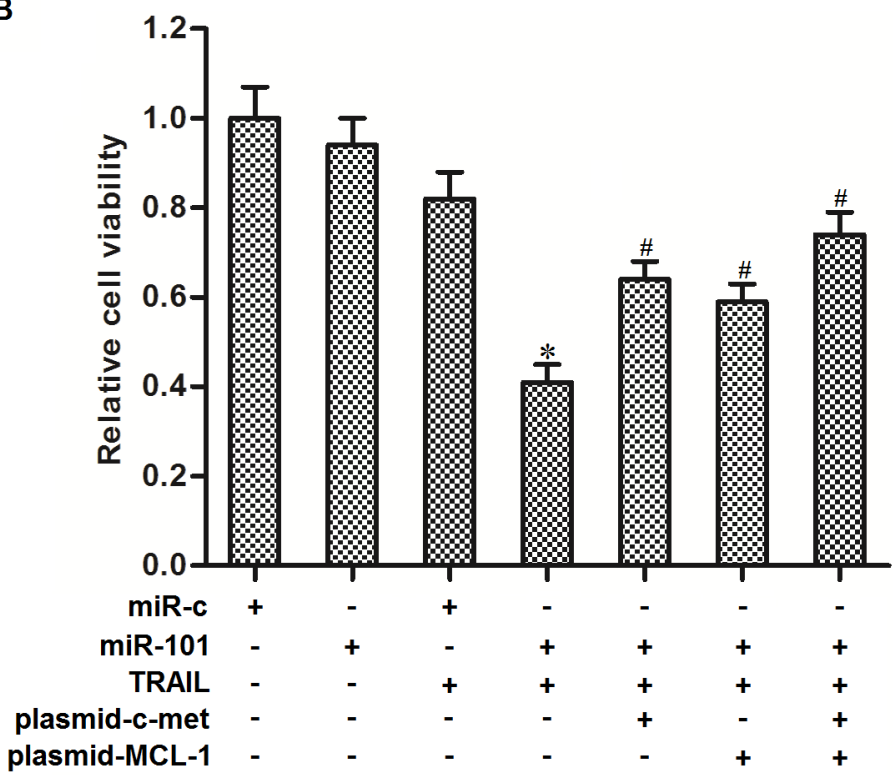

Figure 4: MiR-101 sensitizes TRAIL-induced cell death in TPC-1 by targeting c-met and MCL-1. (A) C-met and MCL-1 plasmid abolished miR-101-induced decrease of c-met and MCL-1 expression, respectively. (B) C-met and MCL-1 plasmid partially inhibited the effect of miR-101 on TRAIL-induced cell death in TPC-1 cells. ${ }^{*} P<0.05$ vs. TRAIL+miR-c group. ${ }^{\#} P<0.05$ vs. TRAIL+miR-101 group. (C) Effect of miR-101, c-met and MCL-1 plasmid on TRAIL-induced activation of caspase- 8 in TPC-1 cells. 


\section{Overexpression of miR-101 enhances anti-tumor effect of TRAIL on PTC in vivo}

To investigate whether miR-101 enhances antitumor effect of TRAIL on PTC in vivo, we established a TPC-1 xenograft model by subcutaneously inoculating with TPC-1 cells which were transfected with lentivirus carrying miR-101 precursor sequence on nude mice. The results of in vivo experiments showed that sizes and weight of TPC-1 xenografts in TRAIL plus miR-101 group were obviously smaller than these in TRAIL single treatment group (Figure 6A). We therefore demonstrated that miR101-overexpressed PTC cells were significantly more sensitive to TRAIL treatment in vivo. Subsequently, we measured the expression of miR-101 and c-met/MCL-1 in purified tumor tissues. We confirmed the overexpression of miR-101 in lentivirus-miR-101-transfected tumors (Figure 6B). Furthermore, the miR-101-overexpressed PTC tumors showed obviously lower protein level of c-met and MCL-1, and lower phosphorylation of PI3K, AKT and Bad (Figure 6C). Token together, we demonstrated that miR-101 decreased the expression of c-met and MCL-1 in PTC both in vitro and in vivo. Overexpression of miR-101 was able to enhance the antitumor effect of TRAIL on PTC.

\section{DISCUSSION}

Drug resistance is a major obstacle for conservative treatment of PTC [26]. Although TRAIL has been found to exhibit anti-tumor effect on PTC, strategies are still required to increase the sensitivity of PTC cells to TRAIL. Recent studies have reported that certain miRNAs regulate TRAIL sensitivity in some cancers $[19,20]$. However, relationship between miRNAs and TRAIL sensitivity in PTC is still unclear. Among these drug-related miRNAs, miR-101 is usually downregulated in cancers. Furthermore, miR-101 is found to sensitize gastric cancer, prostate cancer, bladder
A

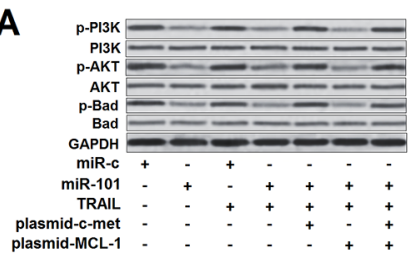

D

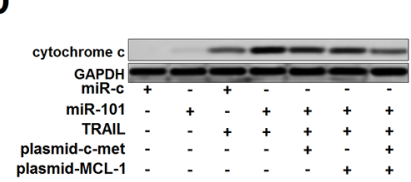

B

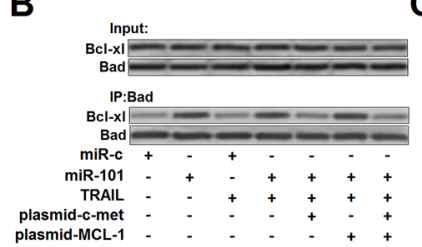

E

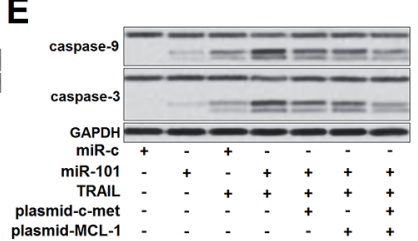

C
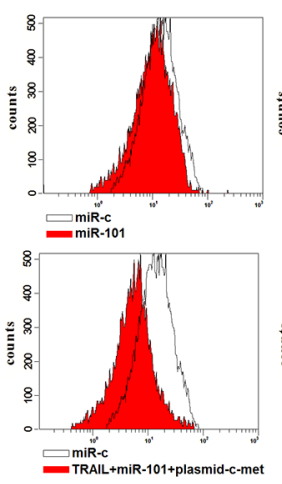

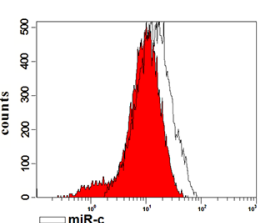

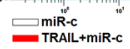

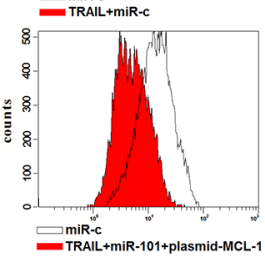

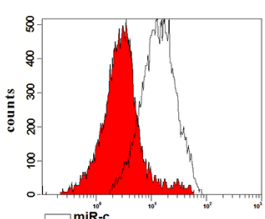

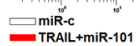

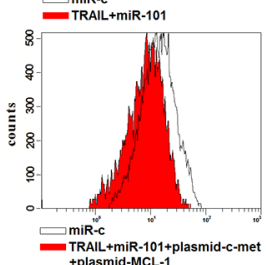

$\mathbf{F}$

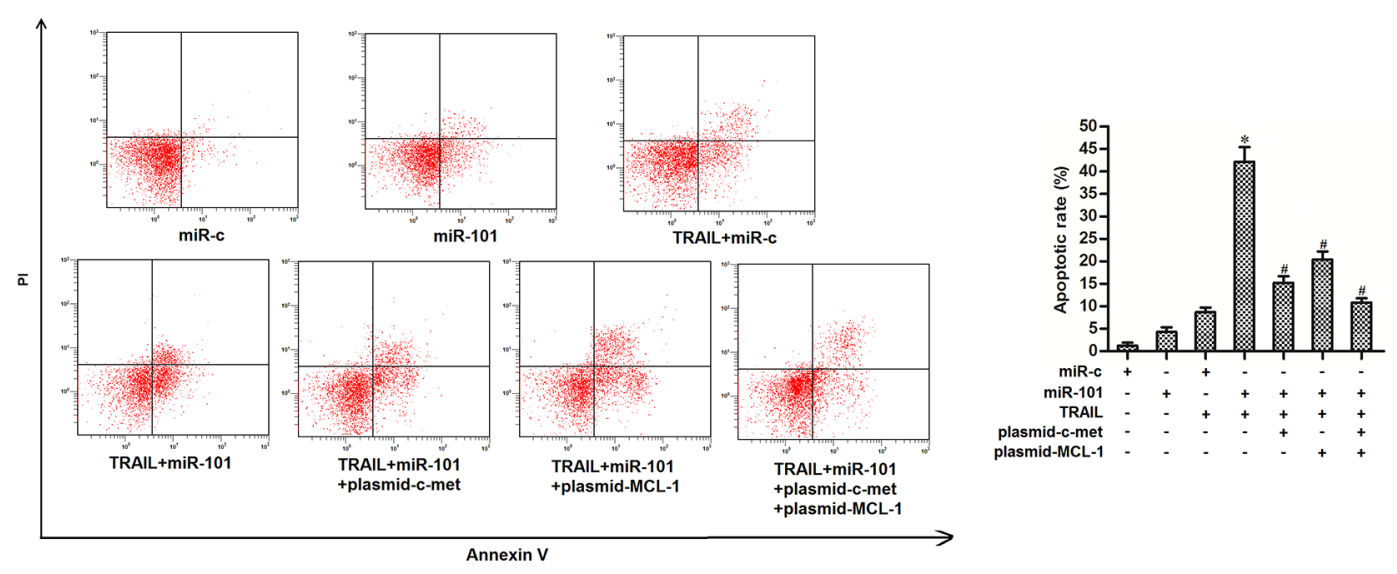

Figure 5: MiR-101 promoted TRAIL-induced mitochondrial apoptosis in TPC-1. (A) TPC-1 cells were transfected with miR-101, c-met and MCL-1 plasmid before they were treated with TRAIL (2 $\mathrm{ng} / \mathrm{ml})$. western blot analysis was performed to evaluate the phosphorylation of PI3K, AKT and Bad. (B) Co-immunoprecipitation was used to examine the effect of miR-101 on changing the interaction with Bad and MCL-1 in TPC-1 cells. (C) TPC-1 cells were transfected with miR-101, c-met and MCL-1 plasmid before they were treated with TRAIL $(2 \mathrm{ng} / \mathrm{ml})$. Flow cytometry analysis was performed to examine the mitochondrial membrane potential. (D) After removal of mitochondria, cytochrome $\mathrm{c}$ in cytoplasm was detected by western blot analysis. (E) After treatment with TRAIL (2 ng/ml), miR-101, c-met and MCL-1 plasmid, activation of caspase-9 and -3 was examined by using western blot analysis. (F) After treatment with TRAIL ( $2 \mathrm{ng} / \mathrm{ml}$ ), miR-101, c-met and MCL-1 plasmid, apoptotic rate of TPC-1 was detected using flow cytometry. ${ }^{*} P<0.05 v s$. TRAIL+miR-c group. ${ }^{*} P<0.05$ vs. TRAIL+miR-101 group. 
cancer and hepatocellular carcinoma cells to anti-tumor drugs $[27,28,29,30]$. These reports demonstrate that miR-
101 is a potential sensitizer for anti-tumor treatment. In the present study, we observed significant decrease of miR-101
A

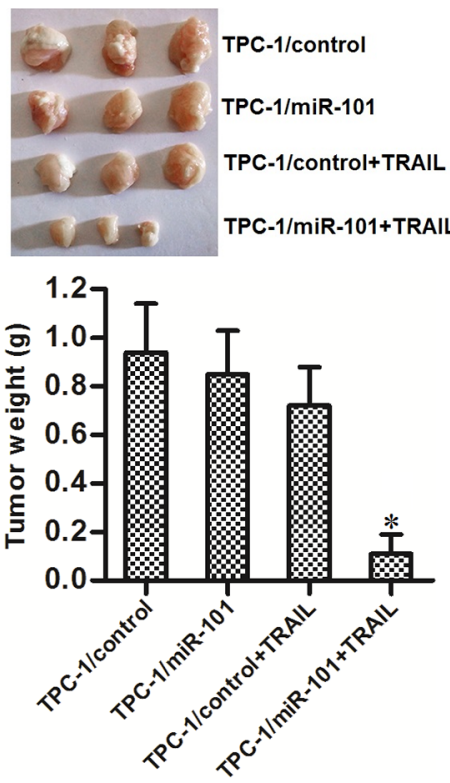

B

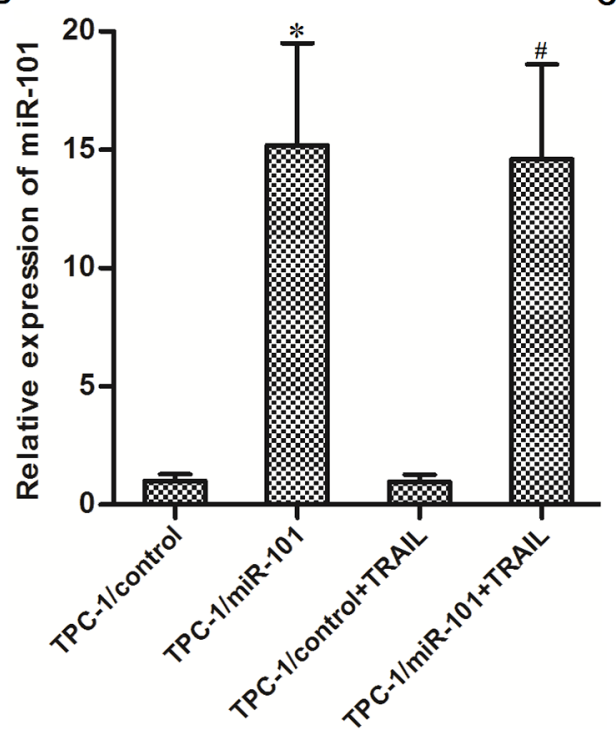

C

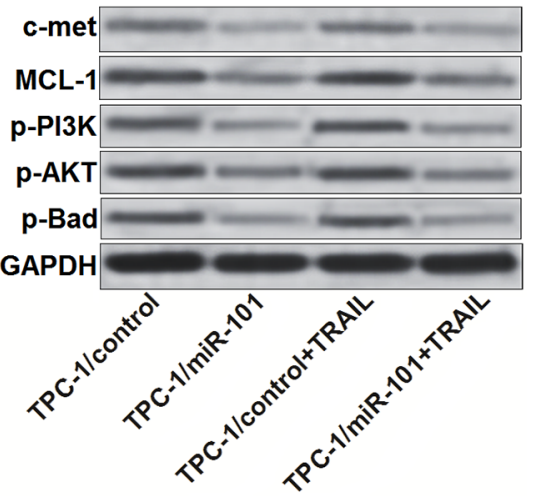

Figure 6: Overexpression of miR-101 enhances anti-tumor effect of TRAIL on PTC in vivo. (A) Nude mice were subcutaneously inoculated with TPC-1/miR-101 cells followed by treating with $40 \mu \mathrm{g} / \mathrm{kg}$ TRAIL per two days. Tumors were purified and weighted after the mice were sacrificed. ${ }^{*} P<0.05 v$ s. TPC-1/control+TRAIL group. (B) Expression level of miR-101 in purified tumors was detected by qRT-PCR analysis. ${ }^{*} P<0.05$ vs. TPC-1/control group. ${ }^{*} P<0.05$ vs. TPC-1/control+TRAIL group. (C) Western blot analysis was performed to detect the protein levels of c-met and MCL-1, and phosphorylation of PI3K, AKT and Bad in purified tumors.

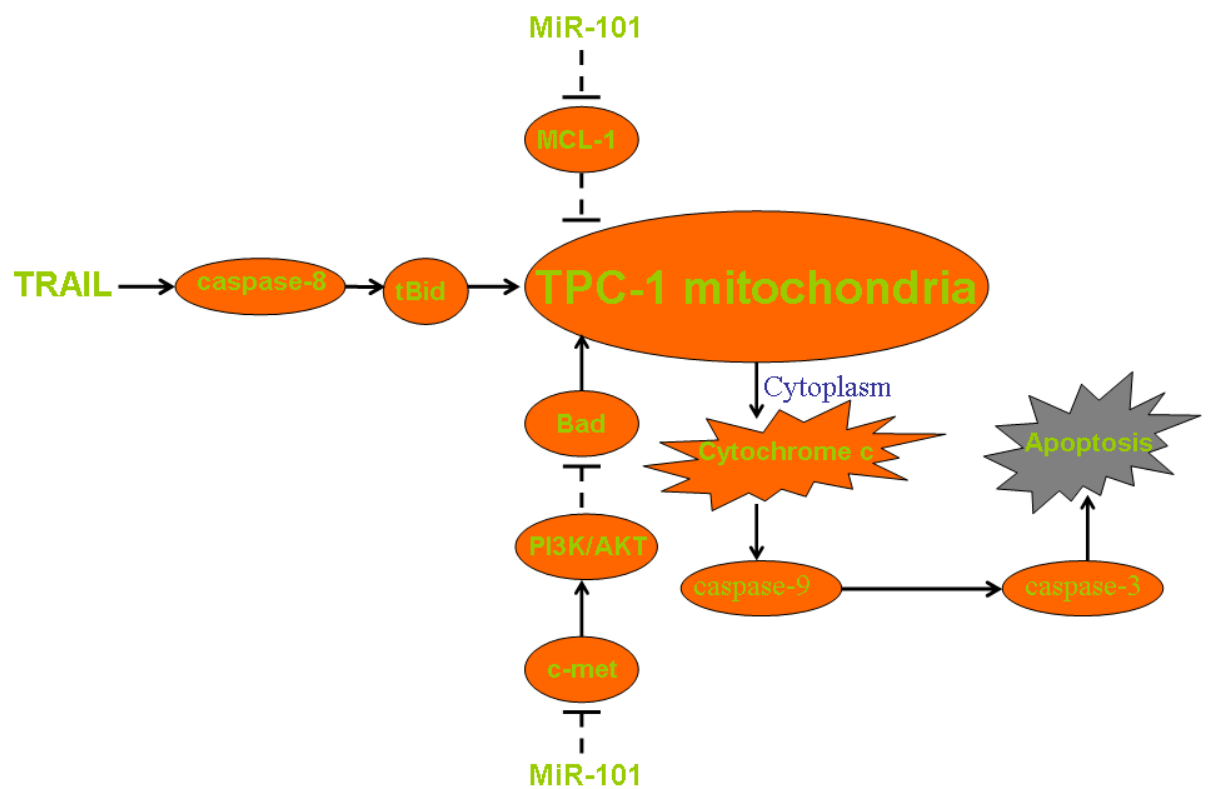

Figure 7: Pathway of mitochondrial apoptosis in miR-101 and TRAIL co-treated TPC-1. MiR-101 decreases the expression of c-met and thus inhibiting the phosphorylation of PI3K and AKT. Low phosphorylation level of AKT induces dephosphorylation of Bad and thus promoting collapse of mitochondria. Besides, miR-101 directly suppresses the expression of MCL-1 to impair the resistance of TPC-1 cells to apoptotic signals. These mechanisms facilitate TRAIL-induced damage of mitochondria, leading to the release of cytochrome $\mathrm{c}$ and the downstream of caspase- 9 activation. Finally, cleaved caspase- 9 triggers caspase-3 and the following apoptosis. 
expression in PTC cells compared to the human normal thyroid epithelial cells. Moreover, evidence was shown that miR-101 was associated with TRAIL sensitivity to PTC. We concluded that overexpression of miR-101 was able to enhance the anti-tumor effect of TRAIL on PTC both in vitro and in vivo.

Hepatocyte growth factor receptor (c-met), also called tyrosine-protein kinase MET, is an oncogene for promoting the tumorigenicity [31]. Abnormal c-met activation is responsible for poor prognosis in cancer patients [32], because activated c-met promotes tumor proliferation, metastasis and formation of new blood vessels [33, 34, 35]. Moreover, reports have proved that overexpression of c-met is correlated with failure of treatment with anti-tumor agents. Among the downstream of c-met pathway, phosphorylation of PI3K and the subsequent activation of AKT represents one key mechanism for formation of anti-apoptosis and drugresistance [36, 37]. Therefore, c-met has been regarded as a promising target for cancer therapy.

Myeloid cell leukemia 1 (MCL-1) is a mitochondrialocated anti-apoptotic protein. It belongs to Bcl-2 family and contains three Bcl-2 homology $(\mathrm{BH})$ domains. MCL-1 can bind to the pro-apoptotic proteins such as Noxa, Puma and Bax, and then inactivate them [38, 39]. Therefore, MCL-1 is an important negative regulator for mitochondrial apoptosis. Indeed, overexpression of MCL1 is revealed to prevent drug-induced apoptotic cell death in multiple cancers. Moreover, knockdown of MCL-1 has been considered as a novel strategy to enhance the effect of anti-tumor drugs including TRAIL [40, 41, 42, 43].

Recently, Studies have reported that both c-met and MCL-1 can be regulated by miRNAs, and the miRNAsc-met/MCL-1 axis determined the drug sensitivity to several cancers $[36,21,44,45]$. In this study, we observed significant overexpression of c-met and MCL-1 in PTC cells. We then showed that expression levels of c-met and MCL-1 were negatively correlated with TRAIL sensitivity in PTC cells. Interestingly, we found that both c-met and MCL-1 can be targeted by miR-101. Restored expression of miR-101 suppressed the functions of MCL-1 and c-met, and thus promoting mitochondrial apoptosis induced by TRAIL.

In summary, we showed the pathway of miR-101-cmet/MCL-1 axis on enhancing TRAIL-induced apoptosis in PTC (Figure 7). Although some PTC cells develop mechanisms against TRAIL-mediated apoptosis, our data provide a potential strategy to increase the sensitivity to TRAIL through introduction with some miRNA sensitizers such as miR-101.

\section{MATERIALS AND METHODS}

\section{Cell culture}

Human normal thyroid epithelial cell line Nthyori3-1 and the PTC cell lines TPC-1, BCPAP and K1 were purchased from the Institute of Biochemistry and Cell Biology, Chinese Academy of Sciences (Shanghai, China). Cells were cultured in Dulbecco's modifed Eagle medium (DMEM, Gibco, Carlsbad, USA) containing with $10 \%$ fetal bovine serum (Gibco) at $37^{\circ} \mathrm{C}$ in a incubator added $5 \% \mathrm{CO}_{2}$.

\section{Detection of miR-101 expression}

Expression of miR-101 in PTC cell lines was detected by quantitative reverse transcriptase real time PCR (qRT-PCR) analysis. Briefly, total RNA was extracted from PTC cells by using the TRIzol reagent (Invitrogen, USA). Subsequently, miR-101 was reverse transcribed by using One Step PrimeScript miRNA cDNA Synthesis Kit (TaKaRa, Dalian, China) and quantified by using SYBR Premix Ex Taq (TaKaRa) under ABI PRISM 7900 Sequence Detection System (Applied Biosystems Prism, USA). Relative expression of miR-101 was normalized to U6 small nuclear RNA (snRNA) and determined by using the $2^{-\triangle \Delta C T}$ analysis method [46].

\section{Transfection}

Control miRNA (miR-c), miR-101 mimics, miR101 inhibitors (anti-miR-101), control siRNA (siRNA-c), c-met small interfering RNA (siRNA-c-met) and MCL-1 small interfering RNA (siRNA-MCL-1) were purchased from GenePharma Co. Ltd. (Shanghai, China). For enforced expression of c-met and MCL-1 in PTC, c-met and MCL-1 eukaryotic expression plasmid (plasmid-cmet and plasmid-MCL-1, respectively) was conducted by cloning the open reading frame of c-met or MCL-1 gene into the pcDNA3.1 plasmid (Life Technologies, USA). RNA oligonucleotides $(50 \mathrm{pmol} / \mathrm{ml})$ or plasmids $(2 \mu \mathrm{g} / \mathrm{ml})$ were transfected into PTC cells by using Lipofectamine 2000 (Invitrogen) according to the manufacturer's instructions followed by incubating for $24 \mathrm{~h}$.

\section{Cell viability assay}

Transfected PTC cells were seeded into 96-well plates overnight before they were incubated with $2 \mathrm{ng} /$ $\mathrm{ml}$ TRAIL for $48 \mathrm{~h}$. Subsequently, MTT assays were performed to measure the cell viability as described previously [47]. The absorbance values of each well were determined at $570 \mathrm{~nm}$ by using a microplate reader (Sunrise Microplate Reader, TECAN, Switzerland). Relative cell viability in experimental groups was normalized to control group.

\section{Luciferase reporter assay}

The wild and mutant 3' UTR of c-met and MCL-1 genes were designed and prepared by GenePharma Co. Ltd. Subsequently, these prepared c-met and MCL-1 3' UTR were cloned into the pGL3 Luciferase Reporter Vectors 
(Promega, USA). For luciferase reporter assay, TPC-1 cells were seeded into 48-well plates overnight, followed by co-transfection with miR-101 (50 pmol/ml), Renilla luciferase $\mathrm{pRL}-\mathrm{TK}$ vector (100 ng/ml, Promega) and pGL3 plasmid with wild/mutant 3' UTR of c-met/MCL-1 gene by using Lipofectamine 2000. $48 \mathrm{~h}$ post-transfection, luciferase assays were performed by using the DualLuciferase Reporter assay system (Promega) according to the manufacturer's instructions. Renilla luciferase activities were normalized to firefly luciferase activities.

\section{Mitochondria removal}

Before western blot analysis on cytochrome c, Mitochondria/Cytosol Fraction Kit (BioVision, USA) was used to separate the mitochondria and cytoplasm fractions in TPC-1 cells. We collected the cytoplasm fraction to detect the protein level of cytochrome $\mathrm{c}$ in following western blot assays.

\section{Immunoprecipitation}

TPC-1 cells were lysed by using RIPA buffer on ice for $30 \mathrm{~min}$ followed by collection of the supernatants. Next, antibody of Bad (Cell Signaling Technologies, USA) was added into the supernatants and incubated overnight at $4{ }^{\circ} \mathrm{C}$. Protein A agarose beads (Cell Signaling Technologies) were then added into the above supernatants and incubated for $2 \mathrm{~h}$ before centrifugation. Subsequently, precipitated agarose beads were washed with cold RIPA buffer, and the proteins binding to the agarose beads were then separated by boiling in sodium dodecyl sulfate (SDS) sample buffer and detected by western blot analysis.

\section{Western blot analysis}

TPC-1 cells were lysed by using RIPA buffer on ice for $30 \mathrm{~min}$ followed by collection of the supernatants. Approximately $50 \mu \mathrm{g}$ of total proteins in the collected supernatants were then separated by $10 \%$ SDS-PAGE followed by transfer to PVDF membranes (Millipore, USA). The membranes were blocked in 5\% skim milk for $1 \mathrm{~h}$ at room temperature and then incubated with the following primary antibodies (Cell Signaling Technologies): anti-c-met, anti-MCL-1, anti-GPADH, anti-caspase-8, -9 and -3 , anti-phosphorylated Bad, PI3K and AKT, anti-Bad, anti-PI3K, anti-AKT, anti-Bcl-xl and anti-cytochrome c. After incubation with primary antibodies overnight, the membranes were washed and incubated with appropriate horseradish peroxidase (HRP)-conjugated secondary antibody for $2 \mathrm{~h}$ at room temperature. Proteins on the membranes were finally probed by using an enhanced chemilu-minescence detection kit (Pierce, USA).

\section{Detection of mitochondrial membrane potential (MMP) and cell apoptosis}

5,5',6,6'-Tetrachloro-1,1',3,3'-tetraethyl imidacarbo cyanine iodide (JC-1, Molecular Probes, USA) and
Annexin V-FITC Apoptosis Detection Kit (Sigma-Aldrich, USA) were used for detection of MMP and cell apoptosis respectively according to the manufacturer's instructions. Both the MMP and cell apoptosis were analyzed by using a flow cytometry (Becton Dickinson, USA).

\section{In vivo tumorigenicity}

Animal care and experimental protocols were approved by the Animal Care Committee of Linyi People's Hospital. For preparation of in vivo experiments, Precusor sequence of miR-101 (5'-UGCCCUGGCUCAGUUAU CACAGUGCUGAUGCUGUCUAUUCUAAAGGUA CAGUACUGUGAUAACUGAAGGAUGGCA-3') was inserted into the lentivirus in Genechem Co., Ltd. $5 \times 10^{5}$ transducing units of recombinant or empty lentivirus were then transfected into the TPC- 1 cells. Subsequently, $5 \times 10^{6}$ of transfected TPC-1 cells were inoculated subcutaneously into the 4-week-old, female nude mice (BALB/c, Shanghai Super-B\&K Laboratory Animal Corp., Ltd., Shanghai, China). For in vivo treatment, TRAIL was administrated by intraperitoneal injection per two days $(40 \mu \mathrm{g} / \mathrm{kg}) .31$ days post-injection, mice were sacrificed before separating the tumors and weighting them. For detection of miR-101, c-met and MCL-1 expression in tumor tissues, collagenase type III was used to purify them.

\section{Statistical analysis}

Experimental data were obtained from at least three times independently experiment. Quantitative data were expressed as the mean \pm SD and analyzed by using SPSS 15.0 software. Differences between groups were compared by using Two-tail Student's t test and ANOVA methods. $P<0.05$ was considered to be statistically significant.

\section{ACKNOWLEDGMENTS}

Thanks are due to the whole contributors who assisted with this study

\section{CONFLICTS OF INTEREST}

The author declared no conflicts of interest.

\section{REFERENCES}

1. Siegel R, Naishadham D, Jemal A. Cancer statistics, 2013. CA Cancer J Clin. 2013; 63:11-30.

2. Nikiforov YE. Thyroid carcinoma: molecular pathways and therapeutic targets. Mod Pathol. 2008; 21:S37-43.

3. Loh KC, Greenspan FS, Gee L, Miller TR, Yeo PP. Pathological tumor-node-metastasis (pTNM) staging for papillary and follicular thyroid carcinomas: a retrospective analysis of 700 patients. J Clin Endocrinol Metab. 1997; $82: 3553-62$. 
4. Sipos JA, Mazzaferri EL. Thyroid cancer epidemiology and prognostic variables. Clin Oncol (R Coll Radiol). 2010; 22:395-404.

5. Deplanque G, Céraline J, Lapouge G, Dufour P, Bergerat JP, Klein-Soyer C. Conflicting effects of caffeine on apoptosis and clonogenic survival of human $\mathrm{K} 1$ thyroid carcinoma cell lines with different $\mathrm{p} 53$ status after exposure to cisplatin or UVc irradiation. Biochem Biophys Res Commun. 2004; 314:1100-06.

6. Giuffrida R, Adamo L, Iannolo G, Vicari L, Giuffrida D, Eramo A, Gulisano M, Memeo L, Conticello C. Resistance of papillary thyroid cancer stem cells to chemotherapy. Oncol Lett. 2016; 12:687-91.

7. LeBlanc HN, Ashkenazi A. Apo2L/TRAIL and its death and decoy receptors. Cell Death Differ. 2003; 10:66-75.

8. Ashkenazi A, Pai RC, Fong S, Leung S, Lawrence DA, Marsters SA, Blackie C, Chang L, McMurtrey AE, Hebert A, DeForge L, Koumenis IL, Lewis D, et al. Safety and antitumor activity of recombinant soluble Apo2 ligand. J Clin Invest. 1999; 104:155-62.

9. Wiley SR, Schooley K, Smolak PJ, Din WS, Huang CP, Nicholl JK, Sutherland GR, Smith TD, Rauch C, Smith CA, Goodwin RG. Identification and characterization of a new member of the TNF family that induces apoptosis. Immunity. 1995; 3:673-82.

10. Mitsiades N, Poulaki V, Tseleni-Balafouta S, Koutras DA, Stamenkovic I. Thyroid carcinoma cells are resistant to FAS-mediated apoptosis but sensitive to tumor necrosis factor-related apoptosis-inducing ligand. Cancer Res. 2000; 60:4122-29.

11. Walczak H, Miller RE, Ariail K, Gliniak B, Griffith TS, Kubin M, Chin W, Jones J, Woodward A, Le T, Smith C, Smolak P, Goodwin RG, et al. Tumoricidal activity of tumor necrosis factor-related apoptosis-inducing ligand in vivo. Nat Med. 1999; 5:157-63.

12. Bu R, Uddin S, Ahmed M, Hussain AR, Alsobhi S, Amin T, Al-Nuaim A, Al-Dayel F, Abubaker J, Bavi P, Al-Kuraya KS. c-Met inhibitor synergizes with tumor necrosis factorrelated apoptosis-induced ligand to induce papillary thyroid carcinoma cell death. Mol Med. 2012; 18:167-77.

13. Cha HY, Lee BS, Chang JW, Park JK, Han JH, Kim YS, Shin YS, Byeon HK, Kim CH. Downregulation of Nrf2 by the combination of TRAIL and Valproic acid induces apoptotic cell death of TRAIL-resistant papillary thyroid cancer cells via suppression of Bcl-xL. Cancer Lett. 2016; 372:65-74.

14. Bartel DP. MicroRNAs: target recognition and regulatory functions. Cell. 2009; 136:215-33.

15. Gargalionis AN, Basdra EK. Insights in microRNAs biology. Curr Top Med Chem. 2013; 13:1493-502.

16. Calin GA, Sevignani C, Dumitru CD, Hyslop T, Noch E, Yendamuri S, Shimizu M, Rattan S, Bullrich F, Negrini M, Croce CM. Human microRNA genes are frequently located at fragile sites and genomic regions involved in cancers. Proc Natl Acad Sci USA. 2004; 101:2999-3004.
17. Hwang HW, Mendell JT. MicroRNAs in cell proliferation, cell death, and tumorigenesis. Br J Cancer. 2006; 94:776-80.

18. Ma J, Dong C, Ji C. MicroRNA and drug resistance. Cancer Gene Ther. 2010; 17:523-31.

19. Sun X, Li Y, Zheng M, Zuo W, Zheng W. MicroRNA-223 Increases the Sensitivity of Triple-Negative Breast Cancer Stem Cells to TRAIL-Induced Apoptosis by Targeting HAX-1. PLoS One. 2016; 11:e0162754.

20. Zhang R, Xu J, Zhao J, Bai J. Knockdown of miR-27a sensitizes colorectal cancer stem cells to TRAIL by promoting the formation of Apaf-1-caspase-9 complex. Oncotarget. 2017; 8:45213-23. https://doi.org/10.18632/ oncotarget.16779.

21. Acunzo M, Visone R, Romano G, Veronese A, Lovat F, Palmieri D, Bottoni A, Garofalo M, Gasparini P, Condorelli G, Chiariello M, Croce CM. miR-130a targets MET and induces TRAIL-sensitivity in NSCLC by downregulating miR-221 and 222. Oncogene. 2012; 31:634-42.

22. Jiang C, Long J, Liu B, Xie X, Kuang M. Mcl-1 Is a Novel Target of miR-26b That Is Associated with the Apoptosis Induced by TRAIL in HCC Cells. Biomed Res Int. 2015; 2015:572738.

23. Brazil DP, Hemmings BA. Ten years of protein kinase B signalling: a hard Akt to follow. Trends Biochem Sci. 2001; 26:657-64.

24. Zeng KW, Wang XM, Ko H, Kwon HC, Cha JW, Yang HO. Hyperoside protects primary rat cortical neurons from neurotoxicity induced by amyloid $\beta$-protein via the $\mathrm{PI} 3 \mathrm{~K} / \mathrm{Akt} / \mathrm{Bad} / \mathrm{Bcl}(\mathrm{XL})$-regulated mitochondrial apoptotic pathway. Eur J Pharmacol. 2011; 672:45-55.

25. Hari Y, Harashima N, Tajima Y, Harada M. Bcl-xL inhibition by molecular-targeting drugs sensitizes human pancreatic cancer cells to TRAIL. Oncotarget. 2015; 6:41902-15. https://doi.org/10.18632/oncotarget.5881.

26. Liu J, Dong BF, Wang PS, Ren PY, Xue S, Zhang XN, Han Z, Chen G. Silencing NOB1 enhances doxorubicin antitumor activity of the papillary thyroid carcinoma in vitro and in vivo. Oncol Rep. 2015; 33:1551-59.

27. Bao J, Xu Y, Wang Q, Zhang J, Li Z, Li D, Li J. miR101 alleviates chemoresistance of gastric cancer cells by targeting ANXA2. Biomed Pharmacother. 2017; 92:1030-37.

28. Huang S, Yang Z, Ma Y, Yang Y, Wang S. miR-101 Enhances Cisplatin-Induced DNA Damage Through Decreasing Nicotinamide Adenine Dinucleotide Phosphate Levels by Directly Repressing Tp53-Induced Glycolysis and Apoptosis Regulator Expression in Prostate Cancer Cells. DNA Cell Biol. 2017; 36:303-10.

29. Lei Y, Li B, Tong S, Qi L, Hu X, Cui Y, Li Z, He W, Zu X, Wang Z, Chen M. miR-101 suppresses vascular endothelial growth factor $\mathrm{C}$ that inhibits migration and invasion and enhances cisplatin chemosensitivity of bladder cancer cells. PLoS One. 2015; 10:e0117809. 
30. He H, Tian W, Chen H, Deng Y. MicroRNA-101 sensitizes hepatocellular carcinoma cells to doxorubicin-induced apoptosis via targeting Mcl-1. Mol Med Rep. 2016; 13:1923-29.

31. Hu CT, Wu JR, Cheng CC, Wu WS. The Therapeutic Targeting of $\mathrm{HGF} / \mathrm{c}-\mathrm{Met}$ Signaling in Hepatocellular Carcinoma: alternative Approaches. Cancers (Basel). 2017; 9:E58.

32. Petterson SA, Dahlrot RH, Hermansen SK, K A Munthe $\mathrm{S}$, Gundesen MT, Wohlleben H, Rasmussen T, Beier CP, Hansen S, Kristensen BW. High levels of c-Met is associated with poor prognosis in glioblastoma. J Neurooncol. 2015; 122:517-27.

33. Daudigeos-Dubus E, Le Dret L, Bawa O, Opolon P, Vievard A, Villa I, Bosq J, Vassal G, Geoerger B. Dual inhibition using cabozantinib overcomes HGF/MET signaling mediated resistance to pan-VEGFR inhibition in orthotopic and metastatic neuroblastoma tumors. Int J Oncol. 2017; 50:203-11.

34. Zhao Y, Xie Z, Lin J, Liu P. MiR-144-3p inhibits cell proliferation and induces apoptosis in multiple myeloma by targeting c-Met. Am J Transl Res. 2017; 9:2437-46.

35. Trovato M, Campennì A, Giovinazzo S, Siracusa M, Ruggeri RM. Hepatocyte Growth Factor/C-Met Axis in Thyroid Cancer: From Diagnostic Biomarker to Therapeutic Target. Biomark Insights. 2017; 12:1177271917701126.

36. Jiang J, Feng X, Zhou W, Wu Y, Yang Y. MiR-128 reverses the gefitinib resistance of the lung cancer stem cells by inhibiting the c-met/PI3K/AKT pathway. Oncotarget. 2016; 7:73188-99. https://doi.org/10.18632/oncotarget.12283.

37. Byeon HK, Na HJ, Yang YJ, Kwon HJ, Chang JW, Ban MJ, Kim WS, Shin DY, Lee EJ, Koh YW, Yoon JH, Choi EC. c-Met-mediated reactivation of PI3K/AKT signaling contributes to insensitivity of BRAF(V600E) mutant thyroid cancer to BRAF inhibition. Mol Carcinog. 2016; 55:1678-1687.

38. Thomas LW, Lam C, Edwards SW. Mcl-1; the molecular regulation of protein function. FEBS Lett. 2010; 584:2981-89.
39. Cory S, Huang DC, Adams JM. The Bcl-2 family: roles in cell survival and oncogenesis. Oncogene. 2003; 22:8590-607.

40. Yu X, Li W, Xia Z, Xie L, Ma X, Liang Q, Liu L, Wang J, Zhou X, Yang Y, Liu H. Targeting MCL-1 sensitizes human esophageal squamous cell carcinoma cells to cisplatininduced apoptosis. BMC Cancer. 2017; 17:449.

41. Woo SM, Min KJ, Seo BR, Kwon TK. YM155 sensitizes TRAIL-induced apoptosis through cathepsin S-dependent down-regulation of Mcl-1 and NF- $\mathrm{KB}-$ mediated downregulation of c-FLIP expression in human renal carcinoma Caki cells. Oncotarget. 2016; 7:61520-32. https://doi. org/10.18632/oncotarget.11137.

42. Park SH, Lee DH, Kim JL, Kim BR, Na YJ, Jo MJ, Jeong YA, Lee SY, Lee SI, Lee YY, Oh SC. Metformin enhances TRAIL-induced apoptosis by Mcl-1 degradation via Mule in colorectal cancer cells. Oncotarget. 2016; 7:59503-18. https://doi.org/10.18632/oncotarget.11147.

43. Kim SY, Park SE, Shim SM, Park S, Kim KK, Jeong SY, Choi EK, Hwang JJ, Jin DH, Chung CD, Kim I. Bay 61-3606 Sensitizes TRAIL-Induced Apoptosis by Downregulating Mcl-1 in Breast Cancer Cells. PLoS One. 2015; 10:e0146073.

44. Xie Q, Wang S, Zhao Y, Zhang Z, Qin C, Yang X. MiR$519 \mathrm{~d}$ impedes cisplatin-resistance in breast cancer stem cells by down-regulating the expression of MCL-1. Oncotarget. 2017; 8:22003-013. https://doi.org/10.18632/ oncotarget. 15781.

45. Cheng D, Xu Y, Sun C, He Z. MicroRNA-451 sensitizes lung cancer cells to cisplatin through regulation of Mcl-1. Mol Cell Biochem. 2016; 423:85-91.

46. Livak KJ, Schmittgen TD. Analysis of relative gene expression data using real-time quantitative PCR and the 2(-Delta Delta C(T)) Method. Methods. 2001; 25:402-08.

47. Tong SJ, Liu J, Wang X, Qu LX. microRNA-181 promotes prostate cancer cell proliferation by regulating DAX-1 expression. Exp Ther Med. 2014; 8:1296-300. 